LA INTEGRACIÓN DEL IMPACTO AMBIENTAL DEL AGUA EN LA INFORMACIÓN FINANCIERA. UNA MIRADA DEL SECTOR HOTELERO DE TUXPAN, VERACRUZ.

\title{
LA INTEGRACIÓN DEL IMPACTO AMBIENTAL DEL AGUA EN LA INFORMACIÓN FINANCIERA. UNA MIRADA DEL SECTOR HOTELERO DE TUXPAN, VERACRUZ
}

\section{WATER ENVIRONMENTAL IMPACT INTEGRATION IN FINANCIAL INFORMATION. AN OVERVIEW OF THE HOTEL SECTOR IN TUXPAN, VERACRUZ}

\author{
Saulo Sinforoso Martínez*, Edalid Álvarez Velázquez**, \\ Berenice Vázquez Patiño***
}

\begin{abstract}
* Doctorado en Ciencias Administrativas y Gestión para el Desarrollo. Profesor de Tiempo Completo de la Facultad de Contaduría de la Universidad Veracruzana, Región Poza Rica-Tuxpan. ORCID: https://orcid.org/0000-0001-6961-5546.

** Doctorado en Gestión Ambiental para el Desarrollo. Directora de la Facultad de Contaduría de la Universidad Veracruzana, Región Poza Rica-Tuxpan. Email: edalvarez@uv.mx. ORCID: https://orcid.org/0000-0002-0251-0376.

*** Estudiante del octavo semestre, Licenciatura en Contaduría de la Facultad de Contaduría, Región Poza Rica-Tuxpan. Email: berenicevazquezpatino@gmail.com. ORCID: https://orcid.org/0000-0001-9746-3666.
\end{abstract}

Dirección para correspondencia: ssinforoso@uv.mx

Fecha de recibido: 10 de julio de 2019

Fecha de aceptación: 8 de agosto de 2019

DOI: https://doi.org/10.19136/hitos.a25n73.3553

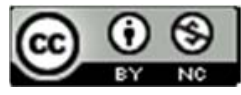


LA INTEGRACIÓN DEL IMPACTO AMBIENTAL DEL AGUA EN LA INFORMACIÓN FINANCIERA. UNA MIRADA DEL SECTOR HOTELERO DE TUXPAN, VERACRUZ.

\section{RESUMEN}

OBJETIVO: Identificar la perspectiva de los directivos de los hoteles de tres estrellas establecidos en Tuxpan Veracruz sobre el reconocimiento contable del impacto ambiental del agua en los Estados Financieros Básicos.

MATERIAL Y METODO: Tiene un enfoque cuantitativo con alcance descriptivo y diseño transversal. Como técnica de recolección de datos se utilizó una encuesta de 11 preguntas cerradas. El estudio se realizó durante el mes de mayo del 2019, en el municipio de Tuxpan de Rodríguez Cano, Veracruz, México. El campo de aplicación fueron los hoteles de tres estrellas, se tomó una muestra por conveniencia de tres hoteles del total de los ocho que existen en el municipio.

RESULTADOS: La mayoría de los encargados de la toma de decisiones dentro de los hoteles de tres estrellas conocen el Costo Ambiental y reconocen que es importante el cuidado del medio ambiente, sin embargo desconocen de un método para reconocer los costos ambientales del agua en los Estados Financieros Básicos.

CONCLUSIONES: Es necesario un programa de difusión, sobre los métodos para el reconocimiento de los Costos Ambientales del Agua en los Estados Financieros Básicos de los hoteles de tres estrellas en el municipio de Tuxpan, Veracruz.

PALABRAS CLAVE: Costo Ambiental. Agua. Estados Financieros Básicos. Costo Sostenible.

\section{ABSTRACT}

OBJECTIVE: To identify hotel managers' perspective in 3-star hotels in Tuxpan, Veracruz; specifically on accounting acknowledgement regarding to water environmental impact in Basic Financial Reports.

MATERIAL AND METHOD: This is a quantitative, descriptive and cross-sectional study. A questionnaire formed by 11 closed questions was administered. This study was carried out in may 
LA INTEGRACIÓN DEL IMPACTO AMBIENTAL DEL AGUA EN LA INFORMACIÓN FINANCIERA. UNA MIRADA DEL SECTOR HOTELERO DE TUXPAN, VERACRUZ.

2019 in Tuxpan de Rodriguez Cano, Veracruz; Mexico. A convenience sample of 3 out of 8 hotels were selected.

RESULTS: Most managers, who make decisions in those 3 star hotels, know about the Environmental Cost and acknowledge the environmental care, however, they are unaware of Hotel Basic Financial Reports in Tuxpan, Veracruz.

CONCLUSIONS: A proper dissemination of information program is needed when it comes to acknowledgement methods on Environmental Water Costs in Basic Financial Reports for 3 starhotels in Tuxpan, Veracruz.

KEYWORDS: Environmental Cost. Water. Basic Financial Statements. Sustainable Cost.

\section{MARCO TEÓRICO}

La necesidad de medir el impacto ambiental, surge cuando comenzó a ser evidente desde el ámbito económico y ecológico que los recursos naturales eran limitados; por una parte, se encuentran los recursos renovables, pero nadie se interesa en mantener un equilibrio en el consumo de estos; y por otro lado, están los recursos no renovables que son explotados de manera desmedida. Los Costos Ambientales tienen antecedentes a nivel internacional desde el año 1948 y nacional desde el año 1971.

Los Costos Ambientales son definidos por Hansen y Mowen (2007, p. 699) como "aquellos en los que se incurre debido a que existe una calidad ambiental deficiente y que están asociados con la creación, la detección, el remedio y la prevención de la degradación ambiental". Estos costos se utilizan para medir el impacto ambiental causado por las actividades económicas de las organizaciones con la finalidad de tomar medidas de prevención.

Sinforoso, Álvarez y González (2018) explican que las "externalidades ambientales deben ser incorporadas en los costos de los bienes o servicios" y se deben registrar a través del Costo Sostenible, los autores utilizan esta cuenta para el registro de las externalidades ambientales, misma que refleja los daños ambientales ocasionados al agua. 
LA INTEGRACIÓN DEL IMPACTO AMBIENTAL DEL AGUA EN LA INFORMACIÓN FINANCIERA. UNA MIRADA DEL SECTOR HOTELERO DE TUXPAN, VERACRUZ.

Díaz (2003, p. 33) menciona que "la empresa en la determinación de sus resultados ignora los efectos sociales y medioambientales por lo que la información dada a través de la Contabilidad Social podría medir su contribución neta a la sociedad".

Parada y Perdomo (2014) describen que "si las organizaciones reconocen contablemente los impactos sociales y ambientales se obtendría una información contable completa que ayudaría a los tomadores de decisiones a contribuir al cuidado y conservación del medio ambiente, pues se tomarían decisiones certeras".

La teoría donde se fundamenta lo expuesto en la presente investigación es, la Teoría del Paradigma del Beneficio Verdadero, Tua (1995) menciona que ésta teoría "se orienta a la medición en abstracto de hechos pasados en búsqueda de un concepto único y autosuficiente de verdad económica". Con el reconocimiento de los Costos Ambientales del Agua en los Estados Financieros Básicos de los hoteles de tres estrellas, en el municipio de Tuxpan de Rodríguez Cano, Veracruz, se genera una informacion financiera completa, buscando beneficios empresariales y ambientales.

De la misma manera, encuentra también su sustento en la teoría de los Stakeholders, con base a Llena (2008) "la Contabilidad y la profesión contable debe colaborar en el cumplimiento de las necesidades de los agentes interesados ofreciendo información y colaborando en la gestión medioambiental por medio de diversas funciones".

Otra de las teorías donde se fundamenta es, la Tridimensional de la Contabilidad, aquella que "se enfoca en la valoración cualitativa y cuantitativa de la existencia y circulación de la riqueza controlada por la organización" (Mejía, Montes y Mora, 2013). El reconocimiento contable del Costo Ambiental del Agua en los Estados Financieros Básicos comprueba esta teoría, pues se integra en Contabilidad el desgaste del recurso natural que utiliza la empresa para brindar sus servicios, surgiendo la necesidad de que las organizaciones se relacionen con conceptos ambientales, que les permita registrar en su información contable los impactos ocasionados a los recursos naturales misma que les permitirá tomar decisiones en beneficio del medio ambiente y la sociedad. 


\section{Original}

LA INTEGRACIÓN DEL IMPACTO AMBIENTAL DEL AGUA EN LA INFORMACIÓN FINANCIERA. UNA MIRADA DEL SECTOR HOTELERO DE TUXPAN, VERACRUZ.

\section{MATERIAL Y MÉTODO}

Tiene un enfoque cuantitativo con alcance descriptivo y diseño transversal. Como técnica de recolección de datos se utilizó una encuesta de 11 preguntas cerradas. El estudio se realizó durante el mes de mayo del 2019 en el municipio de Tuxpan de Rodríguez Cano, Veracruz, México. El campo de aplicación fueron los hoteles de tres estrellas, donde se tomó una muestra por conveniencia de tres hoteles del total de los 8 que existen en el municipio.

\section{RESULTADOS}

En la figura 1, se observa que la mayoría de los encuestados (67\%) tienen conocimiento sobre el concepto de Costo Ambiental.
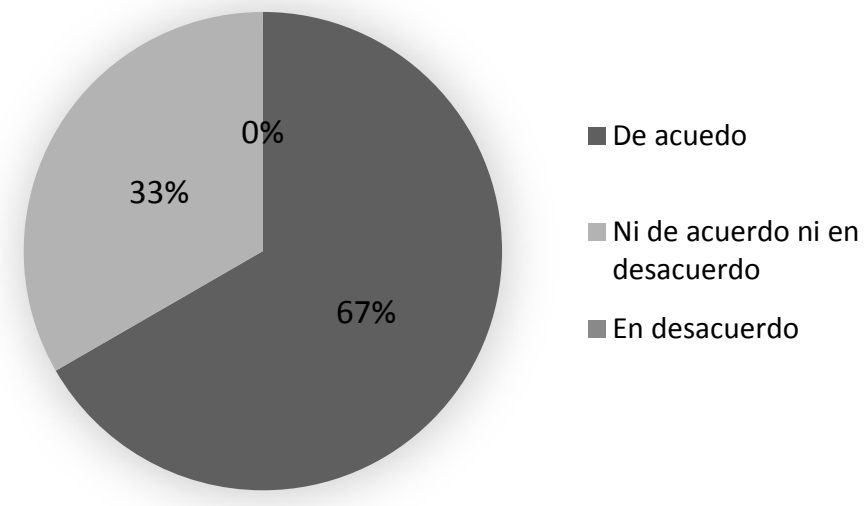

Figura 1. Conocimiento del Costo Ambiental.

Fuente: Elaboración propia.

El 67\% de los encuestados expresó estar de acuerdo en que los recursos naturales repercuten en el periodo de vida de los hoteles, (ver figura 2).

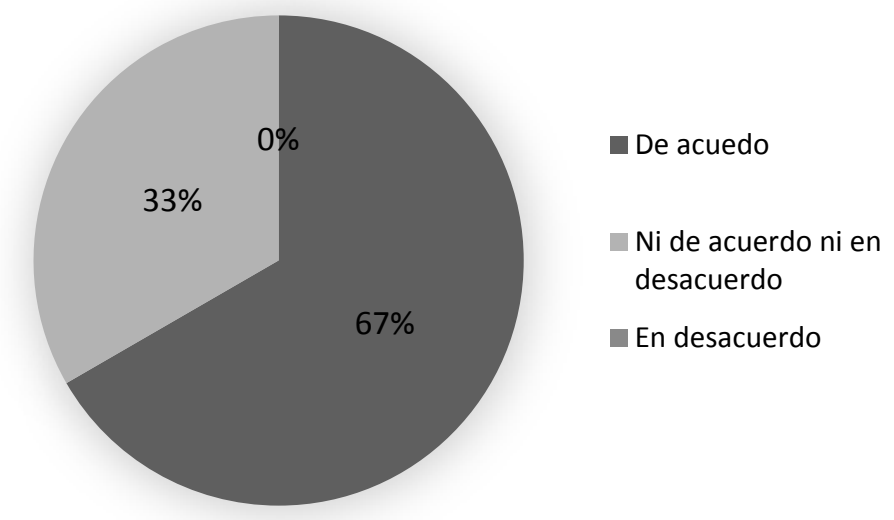

Figura 2. Impacto de los recursos naturales al periodo de vida del servicio de hoteles. Fuente: Elaboración propia. 
LA INTEGRACIÓN DEL IMPACTO AMBIENTAL DEL AGUA EN LA INFORMACIÓN FINANCIERA. UNA MIRADA DEL SECTOR HOTELERO DE TUXPAN, VERACRUZ.

Como puede observarse en la figura 3, el 100\% manifestó estar de acuerdo en que destinar un costo para el cuidado del medio ambiente, genera una ventaja competitiva, expresando la importancia de reconocer el impacto ambiental en Contabilidad.

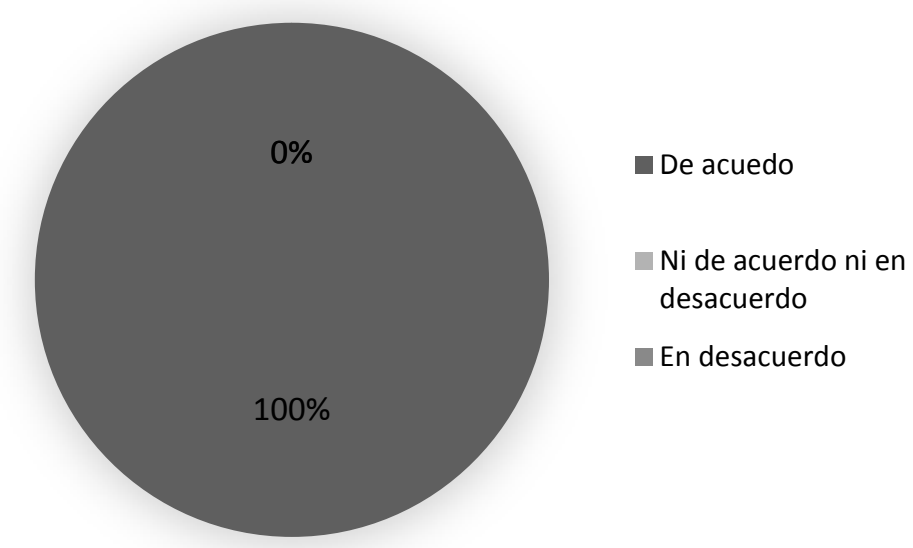

Figura 3. Reconocimiento del impacto ambiental en Contabilidad.

Fuente: Elaboración propia.

La figura 4, muestra que el $67 \%$ de los encuestados no está de acuerdo ni en desacuerdo en integrar un costo en el precio del servicio de hospedaje para destinarlo al cuidado del agua, mientras que el resto (33\%) coincide con la afirmación de Sinforoso, Ricardez y Salazar (2017) al expresar la necesidad de incorporar en el costo de producción o de servicio, los impactos ambientales.

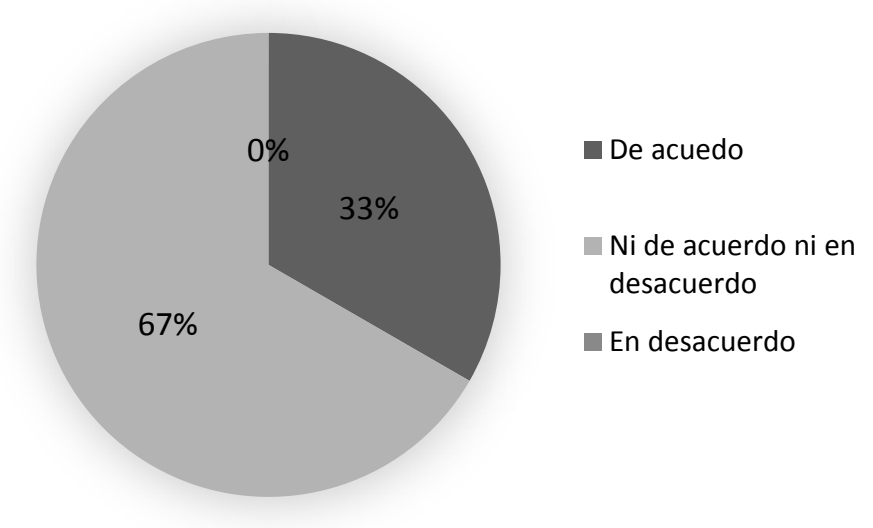

Figura 4. Reconocimiento del Costo Ambiental del agua en el costo del servicio de hotel. Fuente: Elaboración propia. 
LA INTEGRACIÓN DEL IMPACTO AMBIENTAL DEL AGUA EN LA INFORMACIÓN FINANCIERA. UNA MIRADA DEL SECTOR HOTELERO DE TUXPAN, VERACRUZ.

Con base en la figura 5, el 67\% no está de acuerdo ni en desacuerdo en incluir en su precio de venta un costo para reparar los daños ambientales generados por su actividad económica, el resto (33\%) está en desacuerdo.

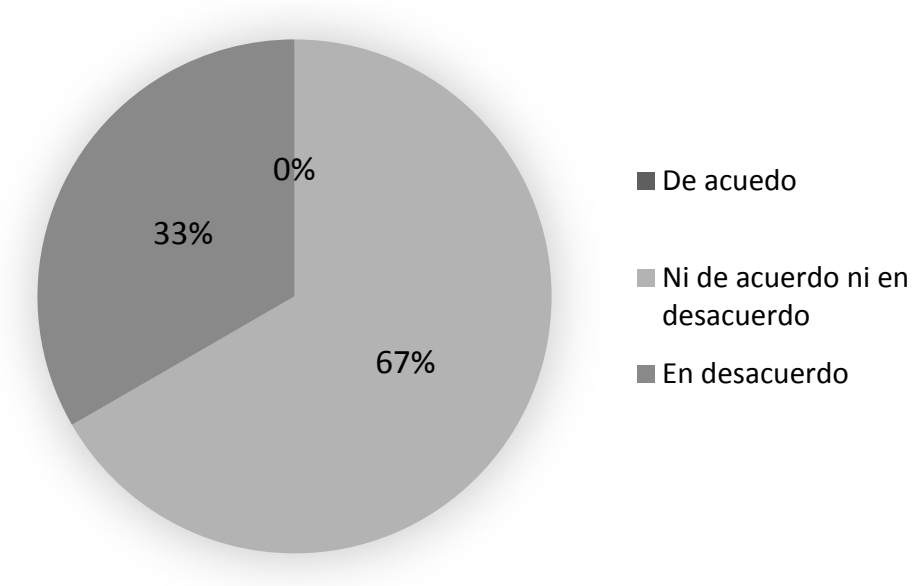

Figura 5. Reconocimiento del Costo Ambiental en el precio de venta.

Fuente: Elaboración propia.

Los datos de la figura 6, reflejan que el 34\% de los hoteles consumen entre 401 a 600 litros de agua al día por la prestación de sus servicios, mientras que el 33\% utiliza entre 601 a 800 litros de agua, el resto (33\%) aplica más de 800 litros.

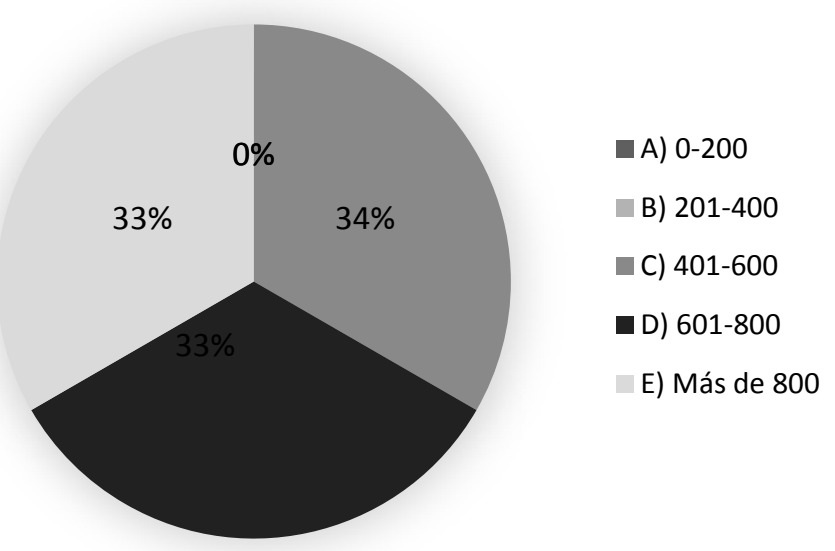

Figura 6. Consumo diario de agua en hoteles de 3 estrellas de Tuxpan Veracruz. Fuente: Elaboración propia. 
LA INTEGRACIÓN DEL IMPACTO AMBIENTAL DEL AGUA EN LA INFORMACIÓN FINANCIERA. UNA MIRADA DEL SECTOR HOTELERO DE TUXPAN, VERACRUZ.

Los valores sirven para el registro de las externalidades ambientales del agua en los Estados Financieros Básicos, mediante la cuenta denominada Costo Sostenible. Tomando como base la investigación realizada por Sinforoso (2018) sobre el reconocimiento contable de las externalidades ambientales en el costo de producción en las empresas cafetaleras Veracruzanas, la presentación del Costo Sostenible en los Estados Financieros Básicos de los hoteles se muestran en las tablas 1, 2, 4 y 5. Además, se utiliza como ejemplo un Costo Sostenible de \$9.00, valor de las externalidades ambientales negativas del agua de los hoteles sujetos de estudio.

Tabla 1

Presentación del Costo Sostenible en el Estado de Resultados Integrales en un hotel de tres estrellas del municipio de Tuxpan de Rodríguez Cano Veracruz

\begin{tabular}{|c|c|c|}
\hline \multicolumn{3}{|c|}{ Hotel “X” } \\
\hline \multicolumn{3}{|c|}{$\begin{array}{l}\text { Estado de Resultados del } 01 \text { al } 31 \text { de Mayo del 20XX } \\
\text { (Cifras en pesos mexicanos) }\end{array}$} \\
\hline Ventas totales & & $\$ 0.00$ \\
\hline (-) Devoluciones y rebajas sobre ventas & & $\$ 0.00$ \\
\hline \multicolumn{3}{|l|}{$=$ Venta netas } \\
\hline (-) Costo de servicio (Costo Sostenible) & & $\$ 9.00$ \\
\hline$=$ Utilidad bruta & & $\$ 0.00$ \\
\hline (-) Gastos de administración & $\$ 0.00$ & \\
\hline (-) Gastos de venta & $\$ 0.00$ & $\$ 0.00$ \\
\hline \multicolumn{3}{|l|}{ (+/-) Resultado Integral de Financiamiento } \\
\hline$=$ Utilidad operativa & & $\$ 0.00$ \\
\hline (-) PTU (10\%) & $\$ 0.00$ & \\
\hline (-) Impuesto Sobre la Renta (30\%) & $\$ 0.00$ & \\
\hline$=$ Utilidad Neta del Ejercicio & & $\$ 0.00$ \\
\hline
\end{tabular}

Fuente: Elaborada con base a la propuesta de Sinforoso (2018).

En la tabla 1, se muestra que el Costo Sostenible forma parte del costo del servicio de las empresas hoteleras y representa un valor económico recuperable al momento de prestar el servicio.

En la tabla 2, se presenta el valor del Costo Sostenible en el Balance General. Cuando un cliente paga el servicio la empresa obtiene un ingreso de $\$ 9.00$ que se representaría en Bancos, pero al 
LA INTEGRACIÓN DEL IMPACTO AMBIENTAL DEL AGUA EN LA INFORMACIÓN FINANCIERA. UNA MIRADA DEL SECTOR HOTELERO DE TUXPAN, VERACRUZ.

mismo tiempo genera un compromiso con el medio ambiente, con base al análisis realizado por Sinforoso, Ricardez y Tapia (2018) sería una Provisión Ambiental, deuda que la empresa generaría con la naturaleza y que sería saldada cuando se implementen actividades en beneficio del medio ambiente financiadas por el recurso obtenido vía Costo Sostenible.

Tabla 2

Presentación del Costo Ambiental del agua mediante el Costo Sostenible en el Balance General Hotel "X"

Balance General del 01 al 31 de Mayo del 20XX

(Cifras en pesos mexicanos)

\begin{tabular}{lllll}
\hline & Activo & & \multicolumn{2}{c}{ Pasivo } \\
Bancos & $\$ 9.00$ & & Provisión ambiental & $\$ 9.00$ \\
\hline
\end{tabular}

Fuente: Elaborada con base a la propuesta de Sinforoso (2018).

Como se observa en la tabla 2, la cuenta de Bancos tiene un monto de $\$ 9.00$, mientras no se aplique este recurso para mitigar el daño ambiental el importe se mantendrá en la cuenta de Provisión Ambiental, partida contable que Sinforoso, Ricárdez y Tapia (2018) proponen al analizar el registro contable del impacto ambiental desde la perspectiva de las Normas de Información Financiera C-9 y las Normas Internacionales de Información Financiera de forma específica en la Norma Internacional de Contabilidad NIC-37.

Al momento de aplicar el recurso obtenido por el Costo Sostenible para el cuidado del agua, la cuenta de Provisión Ambiental desaparece, (ver tabla 3).

Tabla 3

Aplicación de la Provisión Ambiental, 10/06/20XX

\begin{tabular}{lccr}
\hline \multicolumn{1}{c}{ Concepto } & Parcial & Debe & Haber \\
\hline Provisión ambiental & 20 & & \\
$\quad$ Bancos & $\$ 9.00$ & \\
Aplicación del recurso para las externalidades ambientales & & $\$ 9.00$ \\
Sumas iguales & $\$ 9.00$ & $\$ 9.00$ \\
\hline
\end{tabular}

Fuente: Elaborada con base a la propuesta de Sinforoso (2018). 
Original

LA INTEGRACIÓN DEL IMPACTO AMBIENTAL DEL AGUA EN LA INFORMACIÓN FINANCIERA. UNA MIRADA DEL SECTOR HOTELERO DE TUXPAN, VERACRUZ.

En la siguiente tabla, se muestra la representación del Costo Sostenible en el Estado de Flujo de Efectivo.

Tabla 4

Presentación de los Costos Ambientales del agua mediante el Costo Sostenible en el Estado de Flujo de Efectivo Hotel "X"

Estado de flujo de efectivo del 01 al 06 de Junio del 20XX

(Cifras en pesos mexicanos)

\begin{tabular}{ll}
\hline Actividades de operación & Monto \\
\hline Ingreso por el Costo Sostenible & $\$ 9.00$ \\
Pago a empleados & $\$ 0.00$ \\
Pago a proveedores & $\$ 0.00$ \\
Pago a acreedores diversos & $\$ 0.00$ \\
Pago de provisión ambiental & $-\$ 9.00$ \\
Pago de PTU & $\$ 0.00$ \\
Pago de impuestos & $\$ 0.00$ \\
Efectivo generado en operación & $\$ 0.00$ \\
\hline
\end{tabular}

Fuente: Elaboración propia.

Con base a la tabla 4, al cobrar un servicio que incluye impacto ambiental se incrementa el efectivo, al financiar actividades en beneficio del medio ambiente éste disminuye.

Debido a la naturaleza del Costo Sostenible y el efecto que este genera en los recursos de la organización, no impacta en el Estado de Cambios en el Capital Contable ( ver tabla 5), dado que no es un recurso para la empresa, no se generan utilidades; este recurso monetario es una deuda que se tienen con el medio ambiente y que debe ser aplicado para esos fines, confirmando lo que Ázqueta (2002) describe sobre la empresa como el elemento que se encuentra entre el mundo de la economía y el mundo de la biosfera, afirmando que innoven prácticas ambientales con la finalidad de que las futuras generaciones cuenten con recursos naturales para satisfacer sus necesidades, siendo el Costo Sostenible una alternativa. 
Original

LA INTEGRACIÓN DEL IMPACTO AMBIENTAL DEL AGUA EN LA INFORMACIÓN FINANCIERA. UNA MIRADA DEL SECTOR HOTELERO DE TUXPAN, VERACRUZ.

Tabla 5

Presentación de los Costos Ambientales del Agua mediante el Costo Sostenible en el Estado de Cambio en el Capital Contable

Hotel "X"

Estado de Cambio en el Capital Contable del 01 al 06 de junio del 20XX

(Cifras en pesos mexicanos)

\begin{tabular}{lr}
\hline Concepto & Saldos al 06 de Junio del 20XX \\
\hline Capital contribuido & $\$ 0.00$ \\
Capital ganado & $\$ 0.00$ \\
Capital contable & $\$ 0.00$ \\
\hline
\end{tabular}

Fuente: Elaboración propia.

\section{CONCLUSIONES}

Se refleja que es necesario un programa de difusión de la información sobre los métodos para el reconocimiento de los Costos Ambientales del Agua en los Estados Financieros Básicos de los hoteles de tres estrellas en el municipio de Tuxpan de Rodríguez Cano, Veracruz y los beneficios que estos tienen al ser reconocidos en la información financiera.

Se recomienda realizar difusión de la información ante las empresas de diferentes giros, tamaños y actividades económicas sobre los beneficios que tiene el reconocimiento de los Costos Ambientales en los Estados Financieros Básicos, a manera de contribuir con el desarrollo sustentable y la realización de prácticas económicas responsables. Asimismo, mayor concientización por parte de las instituciones educativas, para formar individuos comprometidos con el medio ambiente y a largo plazo formar profesionales socialmente responsables.

Para lograr la integración de los Costos Ambientales en los procesos de producción de las empresas, se recomienda que los Programas Educativos de la Licenciatura en Contaduría integren experiencias educativas pertenecientes a la Contabilidad Social. 
LA INTEGRACIÓN DEL IMPACTO AMBIENTAL DEL AGUA EN LA INFORMACIÓN FINANCIERA. UNA MIRADA DEL SECTOR HOTELERO DE TUXPAN, VERACRUZ.

\section{REFERENCIAS BIBLIOGRÁFICAS}

Azqueta, D. (2002). Introducción a la economía ambiental. España: Mc Graw-Hill Interamericana de España.

Díaz, M. (2003). La contabilidad social: origen y paradigmas. Revistas UNMSM, 10(19), 31- 42. Recuperado de http://sisbib.unmsm.edu.pe/BibVirtual/Publicaciones/quipukamayoc/2003/primer/contabili dad.html

Hansen, D. y Mowen, M. (2007). Administración de costos. Contabilidad y control. Recuperado de http://fullseguridad.net/wp-content/uploads/2016/11/Administracion-de-costos.Contabilidad-y-control-Hansen-5th.pdf

Llena, F. (2008). El papel de la contabilidad en la gestión medioambiental de la empresa: propuestas y evidencia empírica. Revista de Contabilidad y Dirección, 7, 103-134. Recuperado de http://www.accid.org/revista/documents/rcd7_castella_103.pdf.

Mejía, E., Montes, C. y Mora, G. (2013). Estructura conceptual de la teoría tridimensional de la contabilidad. Contexto, 2(1), 49-70. Recuperado de http://revistas.ugca.edu.co/index.php/contexto/article/view/42/338.

Normas de Información Financiera. (2018). Información jurídica inteligente. NIF C-9 Provisiones, contingencias y compromisos. Recuperado de https://doctrina.vlex.com.mx/vid/nif-c-9provisiones-701138929?_ga=2.173852132.690904779.1550448107-

1736730935.1550448107

Normas Internacionales de Contabilidad NIC 37. (2018). Provisiones, activos contingentes y pasivos contingentes. Recuperado de http://normasinternacionalesdecontabilidad.es/nic/pdf/NIC37.pdf

Parada, W. y Perdomo, D. (2014). Contabilidad Social: ¿Una verdadera alternativa? Trabajo presentado en el XIX congreso internacional de contaduría, administración e informática. Ciudad de México: UNAM

Sinforoso, S. (2018). Procedimiento metodológico para el reconocimiento de las externalidades ambientales en el costo de producción de las empresas cafetaleras veracruzanas. (Tesis Doctoral). Universidad Veracruzana, Xalapa, Veracruz. 
LA INTEGRACIÓN DEL IMPACTO AMBIENTAL DEL AGUA EN LA INFORMACIÓN FINANCIERA. UNA MIRADA DEL SECTOR HOTELERO DE TUXPAN, VERACRUZ.

Sinforoso, S, (2018). Reconocimiento Contable de las Externalidades Ambientales en el Costo de Producción. Caso empresas cafetaleras Veracruzanas. En I. Tapia y H. M. Villanueva (Eds.), Paradigmas emergentes de la administración: Innovación, sustentabilidad y emprendimiento social (17-34). Puebla, México: Universidad Iberoamericana Puebla. Recuperado de https://www.researchgate.net/publication/330967386_Reconocimiento_Contable_de_las _Externalidades_Ambientales_en_el_Costo_de_Produccion_caso_empresas_cafetalera s_Veracruzanas

Sinforoso, S., Álvarez, E., y González, T. (2018). Aspectos teóricos del costo sostenible de las organizaciones. En M. B. Flores y F. González (coord.), La gestión como herramienta para el desarrollo empresarial (449-468). Michoacán, México: Ilustre Academia Iberoamericana de Doctores A.C. Recuperado de https://www.researchgate.net/profile/Saulo_Sinforoso_Martinez/publication/328292391_ ASPECTOS_TEORICOS_DEL_COSTO_SOSTENIBLE_DE_LAS_ORGANIZACIONES/Ii nks/5bc4ea01458515f7d9bf122d/ASPECTOS-TEORICOS-DEL-COSTO-SOSTENIBLEDE-LAS-ORGANIZACIONES.pdf

Sinforoso, S., Ricardez, J. y Salazar, F. (2017). Methodology for the accounting recognition of negative environmental externalities in the cafeterium sector companies of the state of Veracruz. Journal of Environmental Accounting and Management, 5 (4), 303-314. DOI 10.5890/JEAM.2017.12.003

Sinforoso, S., Ricárdez, J. y Tapia, O. (Junio de 2018). Análisis del registro contable del impacto ambiental desde la NIF C-9 y la NIC 37. En N. M. Álvarez (Decano), XV conferencia internacional de ciencias económicas, empresariales y jurídicas. Camagüey, Cuba.

Tua, J. (1995). Lecturas de teoría e investigación contable 1. Colombia: Ediciones Gráficas Ltda. 